21st Particles and Nuclei International Conference (PANIC 2017)

International Journal of Modern Physics: Conference Series

Vol. 46 (2018) 1860055 (7 pages)

(C) The Author(s)

DOI: $10.1142 / \mathrm{S} 2010194518600558$

\title{
Tau Identification at the CMS Experiment in LHC Run-2
}

\author{
Somnath Choudhury (for the CMS collaboration) \\ Indian Institute of Science \\ Bangalore, India \\ somnath.choudhury@gmail.com
}

Published 3 May 2018

\begin{abstract}
Since Run-1 of the LHC, CMS has taken the opportunity to improve further particle reconstruction. A number of improvements were made to the hadronic tau reconstruction and identification algorithms. In particular, the reconstruction of the tau decay products leaving deposits in the electromagnetic calorimeter was improved to better model signal of $\pi^{0}$ from $\tau$ decays. This modification improves energy response and removes the tau footprint from isolation area. In addition to this, improvements were made to discriminators that combine isolation and tau lifetime variables, and the rejection of electrons misidentified as hadronic taus was improved using multivariate techniques. The results of these improvements using $13 \mathrm{TeV}$ data at LHC Run- 2 are presented and validation of tau identification using a variety of techniques has been highlighted.
\end{abstract}

Keywords: tau lepton; multivariate isolation; identification efficiency; misidentification rate; trigger.

\section{Introduction}

The $\tau$ lepton, with a mass of $m_{\tau}=1.77 \mathrm{GeV}$, is the only lepton heavy enough to decay into hadrons. In about one third of the cases tau leptons decay leptonically to a muon or an electron with two neutrinos, and are reconstructed and identified with the usual techniques for muons and electrons. In the remaining cases, tau leptons decay hadronically, to a combination of charged and neutral mesons with a tau neutrino. Hadronically decaying tau leptons, denoted by $\tau_{h}$, are reconstructed and identified with the Hadrons plus Strips (HPS) algorithm [1], which was developed for use in the LHC Run-1. The challenge that a tau identification algorithm has to face is the distinction between genuine $\tau_{h}$, and quark and gluon jets, which are copiously produced in QCD multijet process and can be misidentified as $\tau_{h}$. The primary way to reduce the jet $\rightarrow \tau_{h}$ misidentification backgrounds is to use the

This is an Open Access article published by World Scientific Publishing Company. It is distributed under the terms of the Creative Commons Attribution 4.0 (CC-BY) License. Further distribution of this work is permitted, provided the original work is properly cited. 


\section{S. Choudhury}
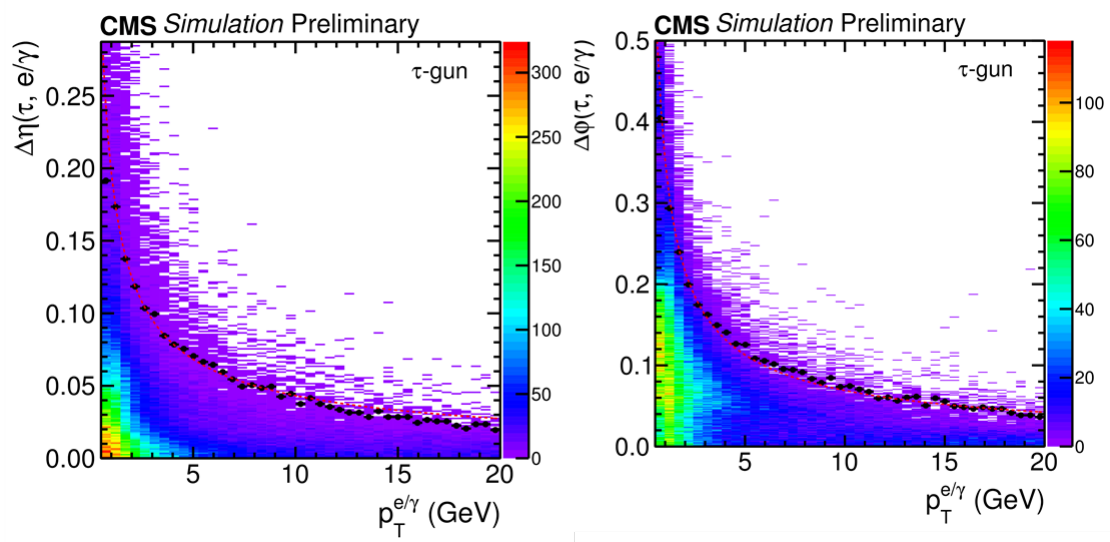

Fig. 1. Strip window size in the $\eta$ (left) and $\phi$ (right) direction as function of the $\mathrm{e} / \gamma \mathrm{p}_{\mathrm{T}}$ for $\tau_{h}$ candidates from simulation. The size of the region in which strips can be reconstructed decreases with $\mathrm{p}_{\mathrm{T}}$. The dashed dots defines the region where $95 \%$ of the $\tau_{h}$ candidates are contained, while the red line is an exponential fit to this function.

fact that the particles produced in $\tau_{h}$ decays are of lower decay product multiplicity, deposit energy in a narrow region compared to a quark or gluon jet, and the primary constituents are typically isolated with respect to other particles in the event. In some final state selections, the misidentification of electrons or muons as $\tau_{h}$ candidates may constitute a sizeable background as well. At CMS, particle resconstruction is performed using the particle-flow (PF) algorithm [2] which combines information from CMS subdetectors to reconstruct and identify particles from each proton-proton collision event. The particles are classified as either charged hadrons, neutral hadrons, photons, muons, or electrons. The particle list from each event are then used to reconstruct the missing transverse energy, jets, the $\tau_{h}$ candidates and to quantify lepton isolation.

\section{Tau Identification}

The $\tau_{h}$ candidates are built from a combination of charged hadron(s) and $\pi^{0} \rightarrow \gamma \gamma$ candidate(s), the latter reconstructed with $\eta-\phi$ clusters of e $/ \gamma$ candidates (electromagnetic strips). Using a set of constraints on the mass and transverse momenta of the decay products of the $\tau$, the HPS algorithm reconstructs the different $\tau_{h}$ hadronic decay modes, notably the $h^{ \pm}, h^{ \pm} \pi^{0}(\mathrm{~s})$, and $h^{ \pm} h^{\mp} h^{ \pm}$signatures. The HPS algorithm used in Run- 2 is similar to the one of Run- 1 , with a few modifications. In particular, a new feature is the dynamic strip reconstruction: the size of the window in which strips are reconstructed is taken as function of the $\mathrm{e} / \gamma$ candidate transverse momentum. This allows for a better containment of the $\tau_{h}$ energy within strips and avoids the associated impact on the isolation energy which is used as a discriminating variable to identify $\tau_{h}$. 
The tau lepton identification in its hadron decay mode is aimed at efficiently selecting the genuine hadronic taus while rejecting the ones misidentified from jets, electrons and muons. After Run-1, additional studies were performed in order to optimize the strip size. In practice, there were cases where $\tau_{h}$ decay products contributed to the isolation, such as: a charged pion from $\tau_{h}$ decay experiences nuclear interaction with tracker material and produces several secondary particles with low $\mathrm{p}_{\mathrm{T}}$. This ends up with low $\mathrm{p}_{\mathrm{T}}$ electrons and photons that go outside strip window. This will affect the isolation of the $\tau_{h}$, although it is part of the $\tau_{h}$ decay product, or photons from $\pi^{0} \rightarrow \gamma \gamma$ have a large probability to convert to an $\mathrm{e}^{+} \mathrm{e}^{-}$pair, and after multiple conversion and bremsstrahlung, electrons and photons may go outside the fixed size window. This will also affect the isolation. If the $\tau_{h}$ has a large $\mathrm{p}_{\mathrm{T}}$ the decay product tend to be boosted in the $\tau_{h}$ flight direction. In this case, a smaller strip size than that considered in Run-1 can reduce background contributions in the strip while accounting for all $\tau_{h}$ decay products. Therefore, a dynamic strip algorithm which is allowed to change in size based on the total $\mathrm{p}_{\mathrm{T}}$ of its constituents has been developed for Run-2. To reject the contribution from misidentified jets, the main handle used is called isolation, which quantifies the activity around the tau candidate: on average it is less for genuine tau than for jets. The cut-based discriminator relies on the isolation sum discriminating variable, which sums the $\mathrm{p}_{\mathrm{T}}$ of particle flow candidates (charged and neutral, excluding the ones that belong to the hadronic tau itself) in a cone centered around the tau axis. Corrections are applied to reduce the effect of pileup on the isolation. Several working points, each targeting a specific signal efficiency, are defined by imposing cuts on the isolation sum variable. The misidentification probability as function of the tau identification efficiency is shown in Fig. 2. An improved multivariate analysis (MVA) based
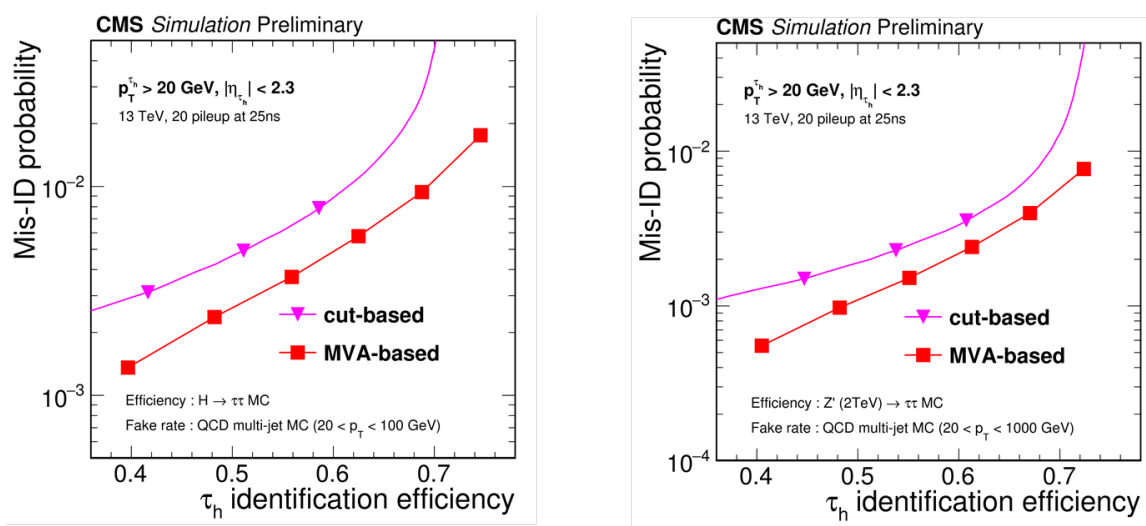

Fig. 2. Misidentification probability, defined as the chance for true QCD jets from simulation to be mis-identified as $\tau_{h}$, as function of the $\tau_{h}$ identification efficiency, evaluated from $\mathrm{H} \rightarrow \tau \tau$ and $\mathrm{Z}^{\prime} \rightarrow \tau \tau$ simulated events. The cut-based discriminant is compared to the MVA-based one. The points correspond to fixed-efficiency working points. 

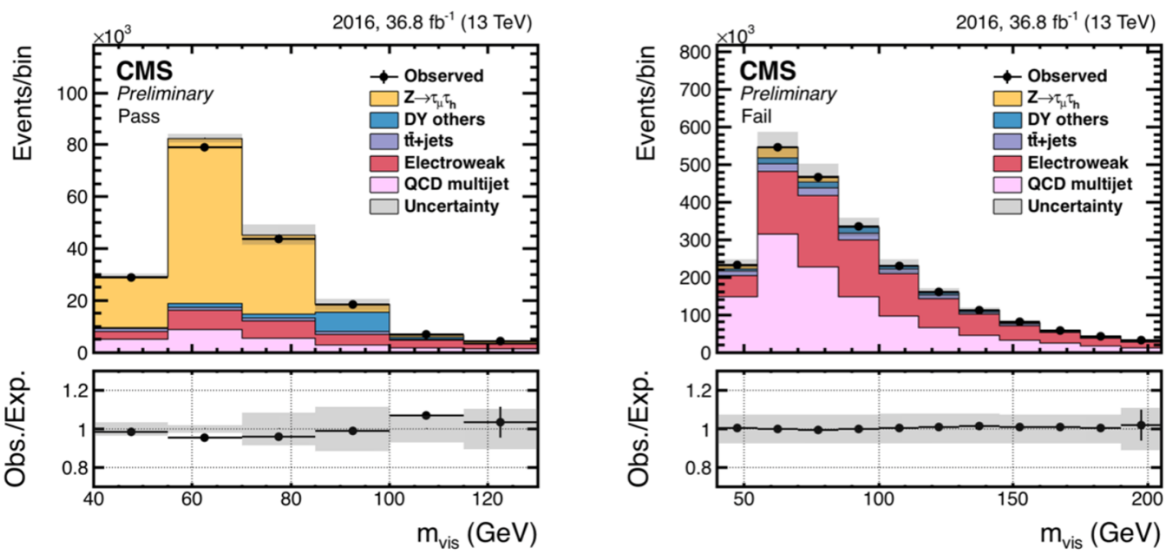

Fig. 3. Postfit distributions in the pass (left) and fail (right) control regions, using the di-tau visible mass as observable, for the loose working point of the MVA-based isolation.

discriminant against jets has also been developed. The use of an MVA approach allows the information from isolation energy, tau lifetime and shower shapes to be optimally combined. With respect to the cut-based approach to isolation, the MVA-based discriminant leads to a significant reduction of the misidentification probability at constant signal efficiency, as demonstrated in Fig. 2.

\section{Tau Identification Efficiency}

The $\tau_{h}$ identification efficiency has been measured in Run-2 data, using three different data-driven techniques [3]: the tag and probe method which relies on $\mathrm{Z} \rightarrow \mu \tau_{h}$ events; the $\mathrm{Z} \rightarrow \mu \tau_{h} / \mathrm{Z} \rightarrow \mu \mu$ ratio method; and using $\mathrm{W}^{*} \rightarrow \tau_{h} \nu$ events. The tag and probe sample of $\mathrm{Z} \rightarrow \mu \tau_{h}$ candidates events comprise at least one well identified and isolated muon (the tag) and one loosely-preselected hadronic tau candidate (the probe). A set of other requirements are imposed to ensure a high purity in $\mathrm{Z} \rightarrow \mu \tau_{h}$. The events are separated into pass and fail categories [4] as shown in Fig. 3, depending on if the $\tau_{h}$ candidate passes the isolation discriminator under study. The signal $\left(\mathrm{Z} \rightarrow \mu \tau_{h}\right)$ and background expected contributions are predicted using simulation, except the QCD multijet and $\mathrm{W}+$ jets backgrounds for which data-driven sideband techniques are used. The parameter of interest, the tau identification scale factor (defined as the ratio of observed data to simulation efficiencies to select $\tau_{h}$ from the $\mathrm{Z} \rightarrow \mu \tau_{h}$ process) is then extracted from a simultaneous maximum likelihood fit in both categories, including systematic uncertainties on the signal and background normalization and shapes. In the fit, the low purity fail category helps to constrain the backgrounds.

\section{Tau Misidentification Probability}

The probability to misidentify quark and gluon jets as $\tau_{h}$ candidates is measured as a function of jet $\mathrm{p}_{\mathrm{T}}$ and using a sample of $\mathrm{W} \rightarrow \mu \nu+$ jet events. The misidentification 
probability is computed as the ratio of the number of jets with $\mathrm{p}_{\mathrm{T}}>20 \mathrm{GeV}$ and $|\eta|<2.4$ that are identified as a $\tau_{h}$ with $\mathrm{p}_{\mathrm{T}}>20 \mathrm{GeV}$ and $|\eta|<2.3$ by one of the tau isolation discriminators to the total number of jets with $\mathrm{p}_{\mathrm{T}}>20 \mathrm{GeV}$ and $|\eta|<2.4$. Note that $\mathrm{p}_{\mathrm{T}}^{\text {jet }}$ differs from the $\mathrm{p}_{\mathrm{T}}$ of the $\tau_{h}$ because the four momentum of the jet is computed by summing the momenta of all its constituents, while the $\tau_{h}$ four momentum is computed only from the charged hadrons and photons included in the decay mode reconstruction of the $\tau_{h}$ candidate. In the measurement of the misidentification probability, backgrounds with genuine $\tau_{h}$ are subtracted based on the expectation from simulated events. The fraction of events with genuine $\tau_{h}$ in the sample passing the $\tau_{h}$ identification criteria is well below $10 \%$ for $\tau_{h}$ with $\mathrm{p}_{\mathrm{T}}<100 \mathrm{GeV}$, but reaches up to $50 \%$ for $\tau_{h}$ with $\mathrm{p}_{\mathrm{T}}$ about $300 \mathrm{GeV}$. Furthermore, backgrounds with prompt muons and electrons giving rise to $\tau_{h}$ candidates are also subtracted based on the expectation from simulated events. To reject events from $\mathrm{Z} / \gamma^{*} \rightarrow \mu \mu$ production a loose anti-muon discriminator is applied to the reconstructed $\tau_{h}$ candidates. The subtraction of backgrounds with real $\tau_{h}$ is subject to an uncertainty of $30 \%$, leading to an uncertainty in the determined jet $\rightarrow \tau_{h}$ misidentification probability of up to $15 \%$. The jet energy scale also leads to a significant uncertainty, in particular in the lowest considered jet $\mathrm{p}_{\mathrm{T}}$ bin because of threshold effects. Additional uncertainties are considered for the probabilities with which electrons are reconstructed as $\tau_{h}$ candidates (100\% relative uncertainty) and with which muons are reconstructed as $\tau_{h}$ candidates and pass the loose working point of the anti-muon discriminator (50\%). These lead to uncertainties in the measured misidentification probabilities of at most a few percent. The observed and simulated jet $\rightarrow \tau_{h}$ misidentification probability [5] for the medium working point of the MVA-based isolation discriminant has been shown in Fig. 4 as functions of $\mathrm{p}_{\mathrm{T}}^{\text {jet }}$ and $\eta^{\text {jet }}$. The observed probabilities show a difference of $10-20 \%$ with respect to the
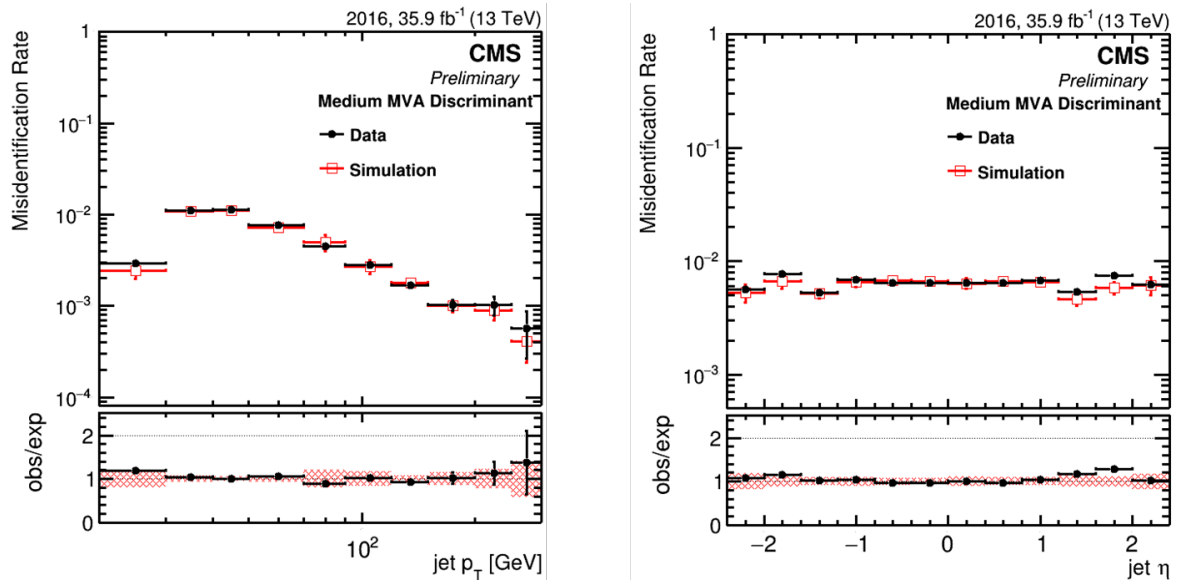

Fig. 4. Misidentification rate of jets to taus versus jet $\mathrm{p}_{\mathrm{T}}$ and $\eta$ for $\mathrm{W}+$ jet events after MVA medium tau identification discriminator: average rate $0.7 \%$ covering wide jet $\mathrm{p}_{\mathrm{T}}$ range $20 \mathrm{GeV}$ $300 \mathrm{GeV}$. 


\section{S. Choudhury}

expectation from simulation. The probabilities were further observed to be almost constant as a function of the pseudorapidity while they decrease monotonically with increasing transverse momentum starting from $\mathrm{p}_{\mathrm{T}}>40 \mathrm{GeV}$ since the absolute isolation increases for quark and gluon initiated jets with growing jet $\mathrm{p}_{\mathrm{T}}$.

\section{Tau Lepton Trigger}

The CMS trigger [6] is organized in two levels: the electronics based Level-1 (L1), which reduces the rate from $40 \mathrm{MHz}$ to $100 \mathrm{kHz}$ and has to take the decision within $3.8 \mu \mathrm{s}$, and a software-based High Level Trigger (HLT) which subsequently reduces the rate from $100 \mathrm{kHz}$ to $1 \mathrm{kHz}$. The reconstruction and selection of $\mathrm{L} 1 \tau_{h}$-trigger objects is based on calorimeter information. At L1 trigger, the basic reconstruction element is the Trigger Tower (TT), which has an extension of roughly $0.087 \times 0.087$ in $\eta$ (pseudorapidity) $\times \phi$ (azimuthal angle), and depending on the position, sums up the energy from the electromagnetic calorimeter (ECAL), hadronic calorimeter (HCAL) or hadronic forward calorimeter (HF). With respect to the previous system, the key conceptual changes to the L1 calorimeter trigger are: all the data from a single event are streamed into one processing card, which allows a global view of the detector and pileup subtraction at L1; and the clustering of the TT energies into L1-objects $\left(\mathrm{e} / \gamma, \tau_{h}\right.$, etc.) is dynamic. In addition, the flexibility of the system allows performing calibration at the TT and L1-object levels, while enabling powerful identification techniques such as isolation to be implemented. The L1 $\tau_{h}$ candidates are built from a collection of TT, which are dynamically clustered around a local energy maximum (seed TT) to capture the $\tau_{h}$ footprint and minimize the effect of pileup. The energy response at L1 with respect to offline for $\tau_{h}$ candidates of 2016 data [7] is represented in Fig. 5.
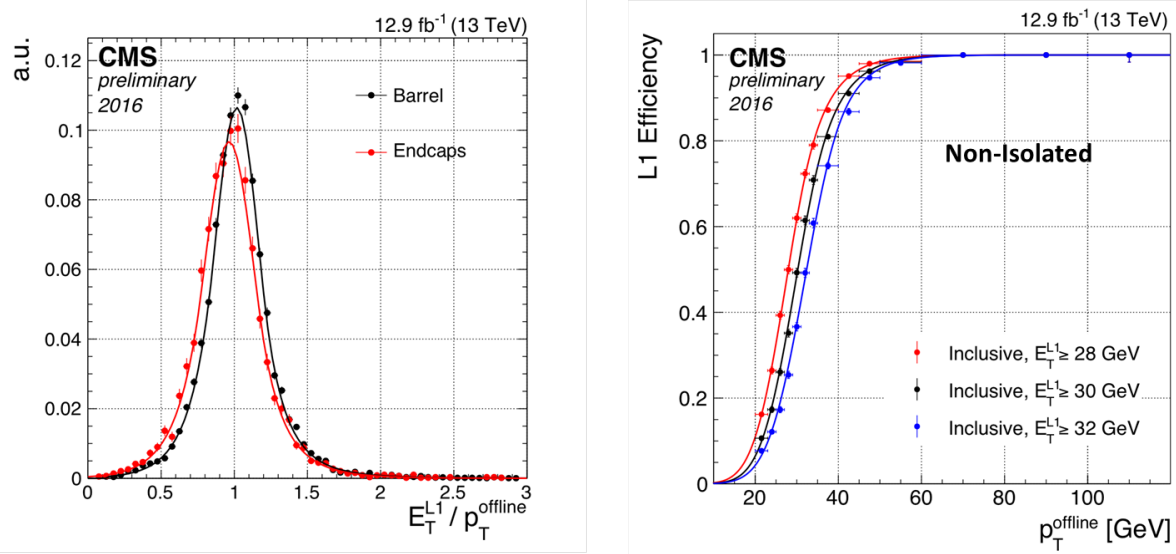

Fig. 5. Energy response of inclusive $\tau_{h}$ seeds for the barrel $\left(\left|\eta^{\text {of fline }}\right|<1.305\right.$, in black $)$ and for the endcaps $\left(\left|\eta^{\text {of fline }}\right|>1.479\right.$, in red) [left]. Level-1 trigger efficiency of non-isolated $\tau_{h}$ seeds as a function of the offline $\tau_{h}$ transverse momentum, for L1 $\mathrm{E}_{\mathrm{T}}$ thresholds of 28,30 and $32 \mathrm{GeV}$. [right] 
The High Level Trigger for $\tau_{h}$ is based on the particle flow algorithm, which performs a global event reconstruction, through an optimal combination of information from the CMS sub-detectors. A list of stable particles (electrons, muons, photons, charged and neutral hadrons) is formed, which are in turn combined to build higher level objects like jets, $\tau_{h}$ and missing transverse energy. At HLT, $\tau_{h}$ is identified combining charged and neutral hadron candidates included in jets reconstructed using the anti- $\mathrm{k}_{\mathrm{T}}$ algorithm with a cone of size $\mathrm{R}=0.4$. Up to three leading charged hadron candidates within a narrow cone (typically $\mathrm{R}=0.1$ ) around the direction of the seeding jet are assumed to be the signature of the decay into charged hadrons. Electromagnetic deposits in ECAL compatible with the $\pi^{0}$ characteristic of $\tau$ decays in $h^{ \pm} \pi^{0}(\mathrm{~s})$ are considered as part of the signature as well. To reduce the contamination from QCD multijets faking $\tau_{h}$, isolation selections are applied to HLT $\tau_{h}$ candidates.

\section{Conclusion}

The Level-1 trigger system of the CMS detector has been recently fully upgraded. Excellent performance in the selection of $\tau_{h}$ at Level-1 has been achieved in 2016 data. The High Level Trigger has also demonstrated good performance, with offinelike reconstruction of $\tau_{h}$. The offline $\tau_{h}$ reconstruction and identification techniques have also been updated in the context of LHC Run-2. In particular, the development of advanced discriminators against jets has been pursued, leading to improved rejection of misidentified hadronic taus. In conclusion, the new tau identification has been shown to have excellent performance in LHC Run-2.

\section{References}

1. The CMS Collaboration, JINST 11, P01019 (2016).

2. The CMS Collaboration, JINST 12, P10003 (2017).

3. The CMS Collaboration, CMS-PAS-TAU-16-002.

4. The CMS Collaboration, CMS DP-2017/006.

5. The CMS Collaboration, CMS DP-2017/036.

6. The CMS Collaboration, CERN-LHCC-2000-038.

7. The CMS Collaboration, CMS DP-2016/044. 